\title{
Aspects of psychomotor development of primary school children with hearing loss from the standpoint of Bernstein's theory of movement construction
}

\author{
Stepanchenko N.I. ${ }^{\mathrm{ABCDE}}$, Hrybovska I.B. ${ }^{\mathrm{CDE}}$, Danylevych M.V. ${ }^{\mathrm{CDE}}$, Hryboskyy R.V. ${ }^{\mathrm{CDE}}$ \\ Lviv State University of Physical Culture named after Ivan Bobersky, Ukraine
}

Author's Contribution: A - Study design; B - Data collection; C - Statistical analysis; D - Manuscript preparation; E - Funds collection.

\begin{abstract}
Purpose:

As the latest research shows, psychomotor development and motor learning of deaf children is garnering a great deal of attention from scientists. Effectively correcting the psychomotor development of hearingimpaired kids requires a deep understanding of the disorders, structures, depth, and the children's potential motor learning capabilities. We believe this understanding can be reached with the Bernstein approach. However, we were able to find only a handful of studies on psychomotor development of hearing-impaired children from the point of view of the theory of how motor skills are formed. Based on this theory, the purpose of this study was to create a diagnostic program that can evaluate and analyze psychomotor disorders and uncover their mechanism in hearing impaired kids compared to their peers with normal hearing.

Material: $\quad$ The study included 54 children from primary schools in Lviv with normal hearing and 94 primary school children with hearing loss from the Lviv Specialized Boarding School named after Maria Pokrova №101 and the Zhovkiv Training and Rehabilitation Center "Zlagoda" (51 children with hearing loss and 43 deaf children). The research sample we chose was random and the children ranged in age from 7-8 years old. To study a single motor skill based on the involvement of multiple levels of central nervous system control, the jump model was chosen. The study consisted of 10 tests, each of which was rated on a 5 point scale. Gathered and analysed data were used for the quantitative method.

Results: $\quad$ It was established that children with hearing loss had psychomotor retardation on all motor skill levels compared to their peers with normal hearing. Hearing impaired children had a low level of static and dynamic coordination, speed of movement, and motor memory. The lowest level of development was purposeful movement.

Conclusions: $\quad$ We identified specific psychomotor disorders in primary school children with hearing loss compared to their peers with normal hearing on all levels of motor skill formation, and found correlations between the overall assessment of hearing impaired children and the studied components by level of movement construction: for any motor activity, there is a primary level, which forms the foundation of the movement and all other levels that are activated when performing the movement. The aspects of sensory-motor functions in primary school children with hearing loss depend on the level of hearing loss and consist of an absence of coordination on multiple levels of movement construction.

Keywords: $\quad$ children, hearing loss, psychomotor, Level-Based Approach, psychomotor deficits.
\end{abstract}

\section{Introduction}

According to the World Health Organization, over 5 $\%$ of the world's population, or 466 million people, has hearing loss (432 million adults and 34 million children). It is estimated that by 2050 , over 900 million people, or 1 in 10 people, will have hearing loss [1]. These figures show that the number of children born with hearing loss is steadily increasing. The problems associated with hearing loss should be addressed early in child development, especially in the early years of life and school years to ensure the child develops properly.

Currently, the process of integrating children with hearing loss is improving due to early diagnostics [2, $3]$, cochlear implants $[4,5]$, as well as early corrective $[6,7]$, and compensatory work [8]. Comprehensive and complete development for hearing impaired children is impossible without physical education, which not only

\footnotetext{
(c) Stepanchenko N.I., Hrybovska I.B., Danylevych M.V.,

Hryboskyy R.V., 2020
}

doi:10.15561/26649837.2020.0308 provides the required level of physical development [9, $10]$, but also corrects deviations in children with auditory deprivation $[11,12]$.

Studies by a number of authors show that children with hearing loss differ in the following aspects of psychomotor development:

- low level of motor skills [13];

- difficulties maintaining static and dynamic equilibrium $[14,15]$;

- relatively low level of spatial orientation development [16];

- $\quad$ slow speed of performance of particular phases and tempos of movements [17];

- deviations in motor skills, which as a whole, characterize a low level of coordinative abilities [18]; relatively low level of figurative thinking development due to a lack of internal speech and verbal support $[19,20]$.

Effectively correcting the psychomotor development 
of young, school-age children with hearing loss requires a deep understanding of the nature of the disorders, their structure, depth, and potential motor development. Such an understanding can only be achieved by studying psychomotor development in the process of applying adequate research techniques.

Motor disorders and pathologies in children have been identified using methods described by Ozeretsky [21], modified by researchers such as: Göllnitz [22] in Germany, Lincoln and Henderson [23] in the USA, and others. Ozeretsky's method is a set of motor skill tests used to study individual components of motion: static coordination, dynamic coordination, speed of movement, rhythm, voluntary muscle movement causing simultaneous involuntary muscle contractions (synkinesis), and simultaneous movements and their forces. It should be noted that there is no generally accepted methodology for studying psychomotor learning. However, in the specialized literature, there are numerous tests with various levels of complexity for studying motor skills in normal-hearing and disabled children: strength, speed, endurance, agility, and flexibility. For children with hearing loss, various holistic techniques and individual quantitative tests are used [24]. However, the quantitative assessment of motor test results does not reveal all the aspects of the motor capabilities of the deaf. A visual description cannot serve as a qualitative analysis, because it does not answer the question: why are children with hearing loss, for example, not able to jump as far as normal-hearing children? Could it be due to muscle weakness in the legs, a low level of neuromuscular coordination, poor motivation, willpower, or other reasons? Using Ozeretsky's method, Weizman [25] noted that some children perform tests intended to evaluate motor skills in older children but fail to perform tests intended for younger children. In other cases, even though subjects were able to perform more complex movements (for example, bouncing a ball on the floor), they had trouble performing what would be considered simple actions, such as throwing a ball forward from their chest.

It is impossible to explain these facts without using Bernstein's Level-Based Approach [26], which can identify the level of the disorder, retardation, and nervous system pathologies, to analyze motor actions and how motor skills tests are structured. All of this prompted us to investigate the motor skills in kids with hearing loss in the context of the level-based theory of movement construction. Based on this theory, this paper attempts to analyze the psychomotor disorders of hearing impaired children and reveal their mechanism - everything from simple movements and actions to complex, deliberate motor skills, to find out the level of motor learning compared to normal-hearing children.

\section{Material and Methods}

Subjects:

The study included normal-hearing children from primary schools in Lviv (54 subjects) and 94 children with hearing impairments - from Lviv Specialized Boarding School named after Maria Pokrova №101 and Zhovkiv Educational and Rehabilitation Center "Zlagoda" (51 subjects with hearing loss and 43 deaf subjects). The research sample we chose was random and the children ranged in ages from 7-8 years old.

Procedure:

The psychological-pedagogical approach to studying psychomotor skills of young, school-age children with hearing impairment includes the use of basic and supplementary methods for a more comprehensive assessment of the development of motor functions. Namely, 1. observational methods: observation of posture, gait, coordination, and facial expressions; 2. studying medical history: with emphasis on the aspects of psychomotor development of children with hearing loss; 3. the metric approach: taking into account Bernstein's theory of movement construction for studying psychomotor development, a method of examining children with hearing loss was developed, consisting of 10 tests. Each test was evaluated using a 5-point scale; 4. methods of mathematical statistics.

For an in-depth study of one motor skill, which is constructed on different levels of central nervous system activity, a jump model was used.

Static motor coordination test:

1. Standing on tiptoes with eyes open and closed before vestibular loading (spinning in place) and after vestibular loading.

The test reveals the predominant activity of the lower level of movement construction (Level A). Stepping out of place or losing balance is considered a mistake.

\section{Dynamic motor coordination tests:}

2. Jump in place, back and forth, right and left, on both feet, and on one foot (test for accuracy and simultaneity of movements and absence of synkinesia).

3. Jump $180^{\circ}$ with hands on waist.

4. Jump and clapping three times in front of the chest while in mid-air.

Tests 2-4 reveal the predominant involvement in the motor skill of the synergy level $B$ and the lower sublevel of the C1 spatial field. They ensure the internal coordination of complex components of one large movement and its correlation with spatial environment.

Tests 2 and 4 are considered incomplete if the subject cannot simultaneously jump with both feet, lands on heels instead of tiptoes, jumps less than 7-8 times within 5 seconds or if the subject makes unnecessary and uncoordinated movements.

Speed of performance tests

5. Jump $180^{\circ}$ while catching a ball upon landing.

6 . Jump in an outlined circle. Any deviations from the circle are recorded.

Tests 5-6 reveal the involvement of the upper sublevel of the C2 spatial field, which determines the maximum accuracy in the final stage of movement. Test 5 is considered incomplete if the subject catches the ball less than three time out of five attempts.

Motor memory and coordination of movement tests: 
7. Jump with various elements of hand coordination (arms up, legs apart and vice versa). Accuracy is recorded.

8. Jump to the rhythm of a light signal. The number of deviations from the rhythm is recorded.

Tests 7-8 reveal the involvement of level $D$ in the hierarchy of movement construction.

Purposefulness of movement tests:

9. The subject must learn to jump half the maximum height they're capable of jumping. After each of the 10 attempts they're given, they are informed of the actual jump height. The test is considered complete if the subject becomes accustomed to jumping half of their maximum height through muscular conditioning.

10. Think of an animal and imitate its movements.

Tests 9-10 reflect a high level of movement construction (level E), which is associated with memorizing the task and conscious self-regulation and improvisation of movements.

If unsuccessful or incomplete, all of the tests can be repeated but no more than three times.

These tests were used to assess the components and levels movement construction for hearing-impaired children. The research methods revealed qualitative disorders of psychomotor skills of children with hearing loss. These tests were not used to identify motor skill development based on the age of the subjects.

Statistical Analyses:

The collected data were analyzed through arithmetic mean, standard deviation, Analysis of Variance (ANOVA), and the measured statistical significance was 0.01-0.05.

\section{Results}

As a result of this psychological-pedagogical study, it was established that compared to their normal-hearing peers, the psychomotor skills of deaf and hard of hearing children are characterized by a lack of development in all of the associated components (Table 1).
Experimentally, it was determined that children with hearing impairment had a lack of static coordination development compared to normal-hearing children (the average score for normal-hearing children was - 4.0; hearing-impaired children -3.0; deaf children - 2.2).

Normal-hearing children had average coordinative motor skills (average score -3.9), while hard of hearing and deaf children had a low level of dynamic coordinative motor skills (hard of hearing - 3.2; deaf-3.0). Compared to their normal-hearing peers, children with hearing impairment had a low level of speed of performance in motor skills (average score for normal hearing - 3.8; hard of hearing -2.6 ; deaf -2.2 ). Motor memory was also found to be on a lower level of development for hearingimpaired and deaf children compared to children with normal hearing (average score for normal hearing - 3.6; hard of hearing -2.4 ; deaf -2.0 ).

The lowest indicators in the study were found to be associated with purposefulness of moment and its meaning on the highest level of movement construction, level $E$, which manifests as memorizing the task at hand, conscious self-regulation, and improvised movements (average score for kids without hearing loss -3.4 ; hard of hearing -2.2 ; deaf -1.6 ). The difference in overall scores for subjects with hearing impairment and deafness compared to peers was 1.3 and 1.9 , respectively.

The results of the study were subjected to mathematical analysis for multiple correlations, which revealed a relationship between the overall assessment of motor development of children with hearing impairment and the studied components by levels of movement construction, characterizing static and dynamic coordination, speed of performance, motor memory and coordination of movement, purposefulness of the movement and its meaning $(r=0.9593)$.

Table 1. Results of the study on psychomotor development in primary school children with deafness, hard of hearing, and normal hearing (in score, mean, standard deviation, and statistical significance)

\begin{tabular}{|c|c|c|c|}
\hline $\begin{array}{l}\text { Parameters for assessment and levels of movement } \\
\text { construction }\end{array}$ & Normal hearing & Hard of hearing & Deaf \\
\hline Static coordination (Level A) & 4.0 & $3.0^{*}$ & $2.2^{* *}$ \\
\hline Accuracy and simultaneity of movements (Level B) & 3.7 & $2.9 * *$ & $1.8^{* *}$ \\
\hline Dynamic coordination (Level C1) & 3.9 & $3.2^{* *}$ & $3.0^{* *}$ \\
\hline Speed of performance (Level C2) & 3.8 & $2.6^{* *}$ & $2.2^{* *}$ \\
\hline $\begin{array}{l}\text { Motor memory and coordination of movements } \\
\text { (Level D) }\end{array}$ & 3.6 & $2.4^{* *}$ & $2.0 * *$ \\
\hline Purposefulness of movement (Level E) & 3.4 & $2.2^{* *}$ & $1.6^{* *}$ \\
\hline Average score for all levels & $3.7 \pm 0.6$ & $2.7 \pm 0.3$ & $2.1 \pm 0.2$ \\
\hline
\end{tabular}

NOTE: Statistical significance: ${ }^{*}-p<0.05 ; * *-p<0.01$ 


\section{Discussion}

Static ataxia observed in children with auditory deprivation is characterized by the difficulty of maintaining balance and the appearance of tremors in the extremities while maintaining a static pose, especially with eyes closed. Deaf children had more difficulty with static coordination than hard of hearing children, who have better vestibular control. According to the data by Melo [15] qualitative characteristics of erect body positioning among deaf children are directly dependent on preservation of vestibular apparatus. Problems with static poses can be explained by a lack of development in regulating movement on level $A$, which provides subconscious, involuntary muscular regulation and arbitrary motor skills, connected with taking a pose and retaining it. Hartman and Vitkovic in their researches $[6,14]$ have identified that deaf children, students of preliminary schools, have difficulties with spatial awareness.

In the course of performing tests on dynamic coordination movements (absence of synkinesia), most subjects with auditory deprivation (deaf children) had uncoordinated movements of the hands and feet. The $180^{\circ}$ jump proved to be the most difficult test for those subjects. In physical education class, the same subjects were observed to have uncoordinated movements while walking and running. Movement features such as shuffling gait, wide foot placement, lateral swing, forward leaning, walking and running on heels and bent legs, and non-linear movements were observed. Melo [27] found the same pathological movement features in his study of deaf children. Increased stiffness and a lack of consistency in movements when performing tests studying the dynamic construction of movement allows us to confirm the presence of synkinesia in children with hearing impairments, which points to a lack of development in level $B$ movement construction - autonomous motor control. We also established a low level of development in the spatial field of sublevel C1, which evaluates the purposefulness of motion. Researches by Engel-Yeger, [13] have shown that hearing and vestibular analyzers dysfunctions are leading towards lowering the level of spatial awareness. This manifests in walking, running, orientation in the body scheme, exercises with objects. This is why the test which required jumping and clapping three times proved rather difficult for the subjects. Fellinger [2] has determined that loss of hearing is also accompanied by delay in motor development.

The majority of subjects showed unnecessary or, conversely, lacked certain movement elements when performing tests on level $D$ of movement construction. Successful completion of the tests were recorded in isolated cases. Therefore, we can state that there is a lack of development in hearing-impaired children on level $D$ - coordinated movement. This is explained by impaired auditory perception, which is caused by specific changes in the decrease of motor memory. This feature was also pointed at by Lévesque [2].

The biggest difference in the compared groups was in the purposefulness of movement (level E). The explanation for this lies in the hearing-impaired children's comprehension of their own voice and other people's voices and their capacity for imaginative thinking. A significant and qualitative difference in the actions of these children is based on the fact that internal speech and language are not activated in the process of solving motor tasks and the ability to plan movements is absent. Additionally, hearing is closely related to movement hearing loss, language development and motor skills are fundamentally interdependent. The absence of internal speech and verbal support causes a delay in motor skill development, what is also shown in researches by Peñeñory [18].

The results reported in this study are in agreement with what has been found in previous studies examining psychomotor skills in the deaf [28, 29].

The analysis of the obtained results allows us to assert that primary school children with hearing loss have delayed development compared to their normal-hearing peers on all levels of movement construction according to Bernstein's theory. This study shows that deafness can lead to problems that may reflect in delayed motor learning and development.

\section{Conclusion}

Bernstein's theory made it possible to isolate the areas of psychomotor impairment in primary school children with auditory deprivation on all levels of movement construction compared to their peers with normal hearing:

- In the hierarchy of the interaction of levels of the central nervous system, which provide levels of movement construction, the principle of dynamic subordination applies. This means that the psychomotor development of kids with hearing loss should be studied from a comprehensive perspective on all levels of movement construction.

- Correlations were established between the general assessment of psychomotor development of primary school children with hearing loss and the studied components by the levels of movement construction, which characterize static and dynamic coordination, speed of performance, motor memory and coordination of movements, and purposefulness of movement. The obtained correlational ties indicate that there are several levels involved in the construction of a particular motor activity: a primary level, which forms the foundation of the movement and all other levels that are activated when performing the movement. The aspects of sensorymotor functions in primary school children with hearing loss depend on the level of hearing loss consist of an absence of coordination on multiple levels of movement construction.

- The analysis of the motor development disorders of primary school children with auditory system pathologies from the standpoint of the level-based theory of movement construction provides an opportunity to study, understand, and choose more appropriate methods of adapted physical education to correct psychomotor retardation. This 
corrective work should be done on an individual basis, taking into account the children's level of psychomotor impairment.

\section{Conflict of interests}

The authors declare that the research was conducted in the absence of any commercial or financial relationships that could be construed as a potential conflict of interest.

\section{References}

1. Deafness and hearing loss [Internet]. 2019 [updated 2019; cited 2019 Nov 03]. Available from: https://www.who.int/ news-room/fact-sheets/detail/deafness-and-hearing-loss

2. Fellinger MJ, Holzinger D, Aigner M, Beitel C, Fellinger J. Motor performance and correlates of mental health in children who are deaf or hard of hearing. Dev. Med. Child Neurol, 2015;57:942-947. https://doi.org/10.1111/dmen.12814

3. Shearer AE, Shen J, Amr S, Morton CC, Smith RJ, Abou Tayoun A, et al. A proposal for comprehensive newborn hearing screening to improve identification of deaf and hard-of-hearing children (vol 21, pg 2614, 2019). Genetics in Medicine. 2019;21(12):2845-2845. https://doi.org/10.1038/s41436-019-0587-x

4. Weaver TS, Shayman CS, Hullar TE. The Effect of Hearing Aids and Cochlear Implants on Balance during Gait. Otol. Neurotol. 2017;38:1327-1332. https://doi.org/10.1097/MAO.0000000000001551

5. Paluch P, Kochanski B, Ganc M, Ciesla K, Milner R, Pluta A, et al. Early general development and central auditory system maturation in children with cochlear implants - A case series. International Journal of Pediatric Otorhinolaryngology. 2019;126. https://doi.org/10.1016/j.ijporl.2019.109625

6. Hartman E, Houwen S, Visscher C. Motor skill performance and sports participation in deaf elementary school children. Adaptedphysicalactivityquarterly:APAQ, 2011;28(2):132-45. https://doi.org/10.1123/apaq.28.2.132

7. Karaush I, Kupriyanova I, Drozdovsky Y, Usov G. Children and adolescents with sensory impairments: problems of depression and suicidal risk. Suicidology. 2017;8(2):70-82.

8. Walowska J, Bolach B, Bolach, E. The influence of Pilates exercises on body balance in the standing position of hearing impaired people. Disability and. Rehabilitation, 2017;13(11):3061-3069. https://doi.org/10.1080/09638288.2017.1370731

9. Winnick JP, Porretta DL. Adapted Physical Education and Sport. Sixth Edition. Champaign, IL: Human Kinetics; 2017.

10.Brice PJ, Strauss G. Deaf adolescents in a hearing world: a review of factors affecting psychosocial adaptation. Adolescent Health Medicine and Therapeutics. 2016;7:67-76. https://doi.org/10.2147/ahmt.s60261

11. Marouli A, Papavasileiou G-E, Dania A, Venetsanou F. Effect of a psychomotor program on the motor proficiency and self-perceptions of preschool children. Journal of Physical Education and Sport, 2016;16(4):1365-1371.

12.Hall ML, Eigsti IM, Bortfeld H, Lillo-Martin D. Auditory Deprivation Does Not Impair Executive Function, But Language Deprivation Might: Evidence From a ParentReport Measure in Deaf Native Signing Children. Journal of Deaf Studies and Deaf Education. 2017;22(1):9-21. https://doi.org/10.1093/deafed/enw054

13.Engel-Yeger B, Weissman D. A comparison of motor abilities and perceived self-efficacy between children with hearing impairments and normal hearing children.
Disability and. Rehabilitation, 2009;31(5):352-358. https://doi.org/10.1080/09638280801896548

14.Vitkovic J, Le C, Lee SL, Clark RA. The contribution of hearing and hearing loss to balance control. Audiol. Neurotol, 2016;21:195-202. https://doi.org/10.1159/000445100.

15.Melo RS, Marinho SE, Freire MEA, Souza RA, Damasceno HAM, Raposo MCF. Static and dynamic balance of children and adolescents with sensorineural hearing loss. Einstein, 2017;15(3):262-268. https://doi.org/10.1590/S1679-45082017AO3976

16.Houde MS, Landry SP, Pagé S, Maheu M, Champoux F. Body Perception and Action Following Deafness. Neural Plasticity, 2016;2016:1-7. https://doi.org/10.1155/2016/5260671

17.LévesqueJ,ThéoretH,ChampouxF.Reduced proceduralmotor learningindeafindividuals. Front. Hum. Neurosci, 2014;8:343. https://doi.org/10.3389/fnhum.2014.00343

18.Peñeñory VM, Manresa-Yee C, Riquelme I, Collazos CA, Fardoun HM. Scoping Review of Systems to Train Psychomotor Skills in Hearing Impaired Children. Sensors (Basel), 2018;18(8):2546. https://doi.org/10.3390/s18082546

19.Yoshinaga-Itano C, Sedey AL, Wiggin M, Chung W. Early hearing detection and vocabulary of children with hearing loss. Pediatrics. 2017;140:e20162964 https://doi.org/10.1542/peds.2016-2964

20.NapoliDJ,MellonNK,NiparkoJK, etal.Shouldalldeafchildren learn sign language? Pediatrics. 2015;136(1):170-176. https://doi.org/10.1542/peds.2014-1632

21.Gurevich MO, Ozeretsky NI. Psychomotor. Technique for the study of motility. Moscow-Leningrad: Gosmedizdat; 1930. (in Russian)

22.Göllnitz G, Schulz-Wulf G. Rhythmic-psychomotor music therapy. A targeted treatment of children and adolescents with developmental problems. Jena: Gustav Fischer; 1976. (In Deutsch)

23.Bruininks RH. Bruininks-Oseretsky Test of Motor Proficiency. Minnesota: American Guidance Service; 1978.

24.Zody JM, Gorman DR. Factorial study of manipulative tests administered to children with deafness ages eight to fifteen. J.Hum.Mov.Stud. 1990;2:85-91.

25. Weizman NP. Psychomotor of oligophrenic children. Moscow: Pedagogy; 1976. (in Russian)

26.Bernshtein NA. The co-ordination and regulation of movements. London: Science; 1967.

27.Melo RS. Gait performance of children and adolescents with sensorineural hearing loss. Gait Posture, 2017;57:109-114. https://doi.org/10.1016/j.gaitpost.2017.05.031

28.Zwierzchowska A, Gawlik K, Grabara M. Deafness and motor abilities level. Biology of Sport, 2008;25(3):263-274.

29.Livingstone N, McPhillips M. Motor skill deficits in children with partial hearing. Developmental Medicine and Child Neurology, 2011;53(9):836-842 . https://doi.org/10.1111/j.1469-8749.2011.04001.x 


\section{Information about the authors:}

Stepanchenko N.I.; (Corresponding Author); Doctor of Pedagogical Sciences, Professor; http://orcid.org/0000-0003-34057024; pp@ldufk.edu.ua; Department of Pedagogy and Psychology, Lviv State University of Physical Culture named after Ivan Bobersky ; 11, Kostiushko, Str., 79007, Lviv, Ukraine.

Hrybovska I.B.; Professor; http://orcid.org/ 0000-0002-0317-2153; recreation@ldufk.edu.ua; Department of Fitness and Recreation, Lviv State University of Physical Culture named after Ivan Bobersky ; 11, Kostiushko, Str., 79007, Lviv, Ukraine.

Danylevych M.V.; Doctor of Pedagogical Sciences; http://orcid.org/0000-0002-1285-392X; recreation@ldufk.edu.ua; Department of Fitness and Recreation, Lviv State University of Physical Culture named after Ivan Bobersky ; 11, Kostiushko, Str., 79007, Lviv, Ukraine.

Hrybovskyy R.V.; http://orcid.org/0000-0003-0398-9152; strilba@ldufk.edu.ua; Department of Shooting and technical sports; Lviv State University of Physical Culture named after Ivan Bobersky ; 11, Kostiushko, Str., 79007, Lviv, Ukraine.

Cite this article as:

Stepanchenko N.I., Hrybovska I.B., Danylevych M.V., Hryboskyy R.V., Aspects of psychomotor development of primary school children with hearing loss from the standpoint of Bernstein's theory of movement construction. Pedagogy of physical culture and sports, 2020;24(3):151-156.

https://doi.org/10.15561/26649837.2020.0308

This is an Open Access article distributed under the terms of the Creative Commons Attribution License, which permits unrestricted use, distribution, and reproduction in any medium, provided the original work is properly cited (http://creativecommons.org/licenses/by/4.0/deed.en).

Received: 05.11.2019

Accepted: 10.12.2019; Published: 05.01.2020 